\title{
The Better Efficiency Calibration for HPGe Detector by Comparing the Single Point Gamma Sources and Multi-nuclides Gamma Source for $\mathbf{k}_{\mathbf{0}}$-NAA Method
}

\author{
A. Yavar ${ }^{\mathrm{a}}$, S. B. Sarmani ${ }^{\mathrm{b}}$, K. S. Khoo ${ }^{\mathrm{a}}$ \\ ${ }^{a}$ School of Applied Physics, Faculty of Science and Technology, Universiti Kebangsaan Malaysia (UKM), \\ 43600 Bangi, Selangor, Malaysia. \\ ${ }^{b}$ School of Chemical Sciences \& Food Technology, Faculty of Science and Technology, Universiti Kebangsaan \\ Malaysia (UKM), 43600 Bangi, Selangor, Malaysia.
}

\begin{abstract}
To obtain high quality of results in gamma spectrometry, it is necessary to calibrate energy and efficiency of gamma detector as accurate as possible. To achieve this aim, utilizing the convenient gamma source can be very useful. Therefore, purpose of present study was to evaluate the determined efficiency by single point gamma sources and multi-nuclides gamma source in a high purity germanium (HPGe) detector. The multi-nuclides source comprised ${ }^{241} \mathrm{Am},{ }^{109} \mathrm{Cd},{ }^{57} \mathrm{Co},{ }^{137} \mathrm{Cs}$ and ${ }^{60} \mathrm{Co}$ nuclides and single point sources of ${ }^{241} \mathrm{Am},{ }^{133} \mathrm{Ba},{ }^{137} \mathrm{Cs}$ and ${ }^{60} \mathrm{Co}$ were utilized under identical geometry to calibrate efficiency of HPGe detector. The source-detector distance was $15.8 \mathrm{~cm}$. The accuracy of the method was evaluated by analyzing IAEA-Soil 7 as certified reference materials in $k_{0}$-neutron activation analysis ( $\left.k_{0}-N A A\right)$ method. Our findings were demonstrated the result of multi-nuclides source was more reliable.
\end{abstract}

Keywords: HPGe detector, Detection efficiency, Point sources, Multi-nuclides source

\section{Introduction}

The use of germanium detectors in high-resolution gamma-ray spectrometry is one of the most widely used procedures for the identification and quantification of unknown gamma-ray emitting radionuclides in samples. It is a non-destructive technique that has the advantage of not requiring laborious sample preparation. This technique requires prior knowledge of the photo-peak efficiency of the detector in the counting geometry for each photon energy. This is usually obtained by an efficiency calibration using certified radioactive source of very similar geometry dimensions, density and chemical composition to the sample that is being studied [1,2].

The calibration of $\gamma$-ray spectrometer includes the energy calibration, and the detection efficiency calibration. The object of energy calibration is to derive a relationship between peak position in the spectrum and the corresponding $\gamma$-ray energy; this is normally performed before measurement. Energy calibration is accomplished by measuring the spectrum of a source emitting $\gamma$-rays of precisely known energy and comparing the measured peak position with energy. The efficiency calibration is to calculate the relationship between number of counts and disintegration rate. This is the parameter of most significance in practical $\gamma$-ray spectrometry. This determination according to the methodology worked out in the $\mathrm{k}_{0}$-instrumental neutron activation analysis ( $\mathrm{k}_{0}$-INAA) method goes as follows [3-6]:

After measurement $\gamma$-ray spectra of source, the peak detection efficiency $\varepsilon_{P}$ is calculated by the following equation:

$$
\varepsilon_{P}=\frac{C P S}{A \cdot \gamma \cdot D \cdot C}
$$

Where, CPS is counts per second of $\gamma$-ray peak of interest, $=\left(\right.$ Net Peak Area $\left./ t_{c}\right)$; A is activity of point source at time manufactured, $A(B q)=C P S / \gamma ; \gamma$ is intensity of $\gamma$-ray emission; $\mathrm{D}$ is decay factor, $=e^{-\lambda t_{d}}$, with $t_{d}$ decay time (from end of irradiation- the date of preparation - to start of counting), and $\lambda=\ln 2 / t_{1 / 2}$ with $t_{1 / 2}$ half-life; $\mathrm{C}$ is counting factor, $=\left[1-e^{-\lambda t_{c}}\right] / \lambda t_{c}$, correcting for decay during counting, with $t_{c}$ counting time [36].

Accuracy of efficiency calibration of detector is necessary to obtain the high precision measurements with radioactive samples. The aim of this study is compare absolute peak efficiency by single point sources and a multi-nuclides source under identical geometry in an HPGe detector. 


\section{Materials And Methods}

From the measurement of calibration sources, experimental efficiencies were calculated. The first step is to choose the "reference" position where source-detector distance is recommended at $15.8 \mathrm{~cm}$ for which the true-coincidence effects are negligible. The sources were counted for 1000 seconds to achieve minimum photopeak fitting errors. For the measurement of full-energy peak detection efficiency $\varepsilon_{P}$ at the selected "reference" position, the multi-nuclides source contained ${ }^{241} \mathrm{Am},{ }^{109} \mathrm{Cd},{ }^{57} \mathrm{Co},{ }^{137} \mathrm{Cs}$ and ${ }^{60} \mathrm{Co}$ was used. Subsequently calculation of $\varepsilon_{P}$ was utilized by single point sources of ${ }^{241} \mathrm{Am},{ }^{133} \mathrm{Ba},{ }^{137} \mathrm{Cs}$ and ${ }^{60} \mathrm{Co}$ under identical geometry. In order to obtain reliable results, one should make use of a source support with centring rings or other means enabling easy and reproducible positioning of the sources versus the detector and for which the distance from source to detector housing can be accurately measured. Preferably, this support (or at least parts of it interposed between the source and the detector) should be made of a material with small gamma-attenuation (e.g. Plexiglas), and the interposed layers should be well-characterized with respect to composition, density and thickness in view of the detection efficiency conversion. The $\gamma$-spectrometry measurements were performed with an HPGe detector (detector model: GC3018, relative efficiency: 30\%) coupled with Canberra Accuspec multichannel analyzer (MCA); the computer code Gamma Acquisition Analysis was used for peak area evaluation. The energy range was from $58.91 \mathrm{keV}$ to $1332.58 \mathrm{keV}$. After the peak detection efficiency values computed by a least square regression analysis, and then the fitted curve is plotted in scale of $\log \varepsilon_{p}$ versus $\log E_{\gamma}[5,6]$.

In order to evaluate the results, IAEA-Soil 7 as certified reference materials was prepared and irradiated in Malaysian Nuclear Agency (MNA) research reactor for 6 hours. Then $\gamma$-spectrometry measurements were found with an HPGe detector and subsequently elemental concentrations in CRMs of IAEA-Soil 7 were calculated by $\mathrm{k}_{0}$-NAA method [6].

The accuracy of the analytical measurements was estimated via the z-score as follows:

$$
z=\frac{\left|C_{i}-C_{r e f, i}\right|}{\sqrt{\sigma_{i}^{2}+\sigma_{r e f, i}^{2}}}
$$

where $C_{i}$ is the concentration of element $i$ in the sample; $C_{r e f, i}$ is the concentration of the certified value for element $i ; \sigma_{i}$ is uncertainty of the concentration of element $i$ in the sample; $\sigma_{r e f, i}$ is uncertainty of the certified respective consensus value for element $i$ [7].

\section{Results And Discussion}

The peak efficiency was calculated using Equation 1. Figure 1illustrations the detection efficiency curve plotted in scale of $\log \varepsilon_{p}$ versus $\log E_{\gamma}$ of an HPGe detector (UKM) for source-detector distance of 15.8 $\mathrm{cm}$. In order to fit the experimental points obtained from multi-nuclides source, the energy was divided into two distinct regions of 58-122 keV, and $122-1333 \mathrm{keV}$ as well as for single point sources, the energy was divided into 58-356 keV, and 356-1333 keV regions. Subsequently, the fit of corresponding polynomials to the energy regions was properly estimated. The corresponding equations of multi-nuclides were $\varepsilon=-10^{-6} E^{2}+0.0002 E-0.0061$ in energy region of $58-122 \mathrm{keV}$ and $\varepsilon=0.1199 E^{-0.694}$ in $122-1333 \mathrm{keV}$, respectively. The corresponding equations of single point sources were $\varepsilon=-2 \cdot 10^{-7} E^{2}+0.00007 E-0.001$ in energy region of 58-356 keV and $\varepsilon=0.2636 E^{-0.831}$ in $356-1333 \mathrm{keV}$, respectively.

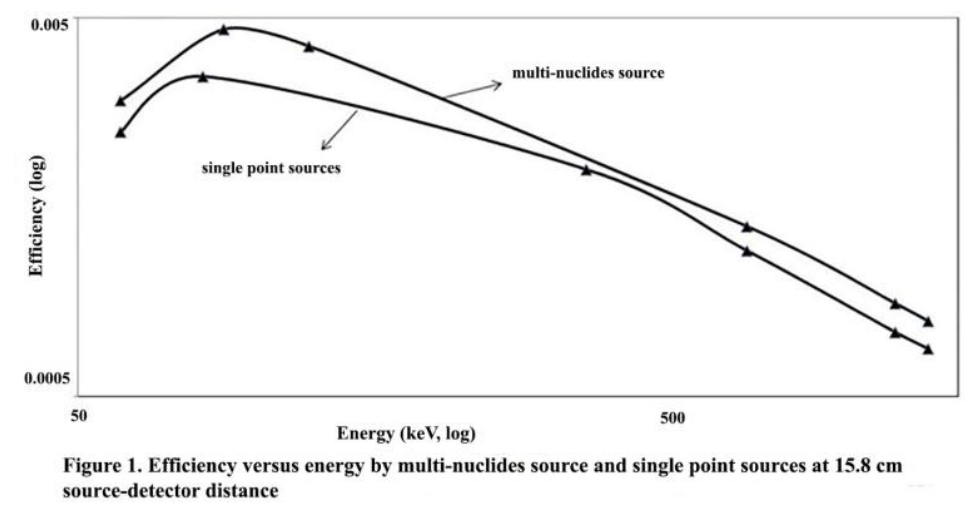


For evaluation of the results, both of the detection efficiency results were used for calculation of elemental concentration of certified reference materials IAEA Soil 7[6]. The comparison between our results and the certified values for the CRMs are presented in Table 1 . The elemental concentrations were calculated by Høgdahl convention. The obtained values show all elemental concentrations were in good agreement to certified values. As well Table 1 shows $z$-score measurements that validate the results for the present study. When using $z$-score (Equation 2), if $z<2$, the result is classified as anticipated; if $2<z<3$, the quality of measurement is "alarming"; and $z>3$, the results are "out of control" [7]. The z-score results for our findings were validated for overall findings $(z<2)$, except $\mathrm{Na}$ value determined using single point sources $(z=7.05)$. Therefore the results of multi-nuclides source have more consistent to certified values, which it indicates using of multi-nuclides source can be useful to obtain more accurate measurements.

Table 1. Comparison of elemental concentrations in experimental results and certified IAEA-Soil 7 by two detection efficiency (single point gamma sources and multi-nuclides gamma source)

\begin{tabular}{|c|c|c|c|c|c|}
\hline Element & $\begin{array}{c}\text { Our work by multi- } \\
\text { nuclides source } \\
(\mathrm{mg} / \mathrm{kg})\end{array}$ & $\begin{array}{c}\text { Our work by single } \\
\text { point sources } \\
(\mathrm{mg} / \mathrm{kg})\end{array}$ & $\begin{array}{l}\text { Certified value } \\
\quad(\mathrm{mg} / \mathrm{kg}\end{array}$ & $\begin{array}{l}\text { z-test of multi- } \\
\text { nuclide } \\
\text { sources } \\
\end{array}$ & $\begin{array}{l}\text { Z-test of single } \\
\text { point sources }\end{array}$ \\
\hline $\mathrm{Eu}$ & $1.01 \pm 0.10$ & $1.26 \pm 0.16$ & $1 \pm 0.10$ & 0.07 & 1.38 \\
\hline $\mathrm{Ga}$ & $10.57 \pm 1.39$ & $11.54 \pm 0.42$ & $10 \pm 1.0$ & 0.33 & 1.42 \\
\hline $\mathrm{La}$ & $27.96 \pm 1.98$ & $29.46 \pm 4.79$ & $28 \pm 0.50$ & 0.02 & 0.30 \\
\hline $\mathrm{Lu}$ & $0.37 \pm 0.15$ & $0.52 \pm 0.31$ & $0.3 \pm 0.08$ & 0.41 & 0.69 \\
\hline $\mathrm{Na}$ & $2382 \pm 36$ & $2984 \pm 66$ & $2400 \pm 50$ & 0.29 & 7.05 \\
\hline
\end{tabular}

\section{Conclusion}

To obtain reliable measurements of radionuclide activity, the knowledge of the detector absolute peak efficiency in the counting conditions is required. The detection efficiency of HPGe detector was determined by single point source and multi-nuclides source under identical geometry. The obtained results indicate which using the multi-nuclides source was more accurate.

\section{Acknowledgments}

The authors would like to thank the Ministry of Education Malaysia for financial support of fundamental research grant scheme under the project code UKM-ST-07-FRGS0235-2010.

\section{References}

[1] Lenn F. Knoll. 1989. Radiation detection and measurement. John Wiley \& Sons, Second edition.

[2] Moens, L., De Donder, J., Lin, X., De Corte, F., De Wispelaere, A., Simonits, A. and Hoste, J. 1981. Calculation of the absolute peak efficiency of gamma-ray detectors for different counting geometries. Nuclear Instrumental Methods. 187, 451-472.

[3] Harb, S., Salahel Din, K. and abbady, A. Study of Efficiency Calibrations of HPGe Detectors for Radioactivity Measurements of Environmental Samples. Proceedings of the $3^{\text {rd }}$ Environmental Physics Conference, 19-23 Feb. 2008, Aswan, Egypt.

[4] Loat, B.V.,Quan, N.V., Anh, L.T.,Anh, T.T.,Nghia, N.T.and Hung, N.V. 2009. Studying of characteristic of GEM40P4 HPGE detector by experiment. VNU Journal of Science, Mathematics - Physics. 25, 231-236.

[5] Vukanac, I.,Djurasevic, M., Kandic, A., Novkovic, D., Nadjerdj, L. andMilosevic, Z. 2008. Experimental determination of the HPGe spectrometer efficiency curve. Applied Radiation and Isotopes. 66, 792

[6] Yavar, A.R., Sarmani, S.B., Wood, A.K., Zainal, N. S. and Khoo, K.S. 2011. Development and implementation of Høgdahl convention and Westcott formalism for $\mathrm{k}_{0}$-INAA application at Malaysian Nuclear Agency reactor. Journal of Radioanalytical and Nuclear Chemistry. 291(2): 521-527.

[7] Yavar, A.R., Khalafi, H., Kasesaz, Y., Sarmani, S.B., Wood, A.K. \& Khoo, K.S. 2012. Verification of MCNP simulation for neutron parameters measurement at Malaysian nuclear agency research reactor. Applied Radiation and Isotopes. 70(10): 24882493. 